\title{
Theoretical Validation of Inheritance Metrics for Object-Oriented Design against Briand's Property
}

\author{
Kumar Rajnish \\ Birla Institute of Technology, Mesra, Ranchi-835215, Jharkhand, India \\ Email: krajnish@bitmesra.ac.in
}

\begin{abstract}
Many inheritance metrics can be found in the literature, but most of those are validated theoretically by using Weyuker's property. Theoretical validation of inheritance metrics using Briand's property is rare in the literature. This paper considers the metrics proposed by Rajnish and Sandip and presents a theoretical validation of the inheritance metrics using the Briand's size and length properties of an inheritance hierarchy. This paper also gives the projection and viewpoint of the inheritance metrics.
\end{abstract}

Index Terms - Object-Oriented, Inheritance Metrics, Briand Properties, Complexity, Classes.

\section{INTRODUCTION}

Nowadays, the uses of Object-Oriented (OO) software metrics have increased rapidly. So, software metrics is the essential to measure the software quality factors. A good software metrics should be validated for its use. In order to provide mathematical rigour and an axiomatic basis to metrics, necessary properties have been set forth by Weyuker [1] and Briand [2] against which metric proposals can be evaluated. Few of the inheritance metrics have been validated using Briand properties. Evaluation of metrics against Briand measurement concepts provides insights into the characteristics and defects of the metrics. Besides serving as a tool for classification and understanding, property based evaluation can also potentially lead to formulation of new metrics. Sandip et al [3] [4] and Rajnish et al [5] [6] proposed four Inheritance metrics and validated using Weyuker properties. This paper shows the validation of the metrics using Briand properties.

For OO systems, most of the inheritance metrics have been validated theoretically by Krishna et al [7] and Joshi et al [8] using Briand properties. Metrics exist for measurement of inheritance at the higher levels of abstraction in OO systems has been given in [3-6]. Rajnish had presents a new class complexity metric of an OO program which is used to predict the understandability of classes in software projects. Their propose complexity metric is evaluated theoretically against Weyuker's properties to analyze the nature of metric and empirically evaluated [9].

Various inheritance metrics have been proposed and their reviews are available in the literature. Chidamber and Kemerer [10] proposed the DIT metric, which is the length of the longest path from a class to the root in the inheritance hierarchy and the NOC metric, which is the number of classes that directly inherit from a given class. Henderson-Sellers [11] suggested the AID (average inheritance depth) metric, which is the mean depth of inheritance tree and is an extension of Chidamber and Kemerer DIT. Li [12] suggested the NAC (number of ancestor classes) metric to measure how many classes may potentially affect the design of the class because of inheritance and NDC (number of descendent classes) metric to measure how many descendent classes the class may affect because of inheritance. $\mathrm{Li}$ [12] also theoretically validated Chidamber and Kemerer metrics using a metric evaluation framework proposed by Kitchenham et al [13] and discovered some of the deficiencies of Chidamber and Kemerer metrics in the evaluation process and proposed a new suite of $\mathrm{OO}$ metrics that overcome these deficiencies. Tegarden et al. [14] proposed the CLD (class-to-leaf depth) metric, which is the maximum number of levels in the hierarchy that are below the class and the NOA (number of ancestor) metric, which is the number of classes that a given class directly or indirectly inherits from. Lake and Cook [15] suggested the NOP (number of parents) metric, which is the number of classes that a given class directly inherits from and the NOD (number of descendants) metric, which is the number of classes that directly or indirectly inherit from a class. Alshayeb et al [16] empirically validated two different software processes. Agarwal et al [17-18] described the approach of empirical study of OO metrics and presented OO design metrics.

The rest of the paper is organized as follows. Section II describes Briand's length and size properties for validating inheritance metrics. Inheritance metrics have been taken for evaluation is described in section III. Section IV describes viewpoint and projection of inheritance metrics presented by Rajnish and Sandip. Section V provides theoretical validation of inheritance metrics against Briand's properties. Section VI presents Conclusion and Future work respectively.

\section{BRIAND’S SIZE AND LENGTH PROPERTY}

\section{A. Size Properties}

Size metrics are commonly found in $\mathrm{OO}$ approaches. Sizes are not bounded, and they are computed as positive integers. The three size properties namely non-negativity, 
presence of null value and module additivity as outlined by Briand et al. [2] are summarized in Table 1.

\section{B. Length Properties}

Length of a system is seen as size of the shortest path between two extremes of the system. Length is therefore

Table 1. Size Properties of Briand

\begin{tabular}{|c|l|}
\hline Property & \multicolumn{1}{|c|}{ Description } \\
\hline S1 (Non-negativity) & The size of a system is nonnegative. \\
\hline S2 (Null value) & $\begin{array}{l}\text { The size of a system is } 0 \text { if the set of } \\
\text { elements which constitute the system } \\
\text { is empty. }\end{array}$ \\
\hline S3(Module Additivity) & $\begin{array}{l}\text { The size of a system cannot be more } \\
\text { than the sum of the sizes of its } \\
\text { modules. In the case of disjoint } \\
\text { modules, the size of a system is equal } \\
\text { to the sum of the sizes of the modules. }\end{array}$ \\
\hline
\end{tabular}

not the same as size, since it captures the size from the point of view of the extreme limits, whereas, size in general captures the measured as a whole. Lines of Code and CLD are examples of size and length metrics respectively. The length properties observed by Briand et al. [1] are summarized in Table 2.

Length properties $L 1$ and $L 2$ are the same as $S 1$ and $S 2$ respectively. Size metrics satisfy non-increasing monotonicity (L3) and non-decreasing monotonicity property (L4), since adding relationships between the elements within a module or between the elements of different modules in the system does not change the count of the entities already present in the system. However, the considered size metrics do not satisfy module merger (L5) because the size metrics are sum oriented and not comparison oriented. Thus, it can be observed that metrics satisfying sum oriented size property $S 3$ do not satisfy comparison oriented length property L5.

Table 2. Length Properties of Briand

\begin{tabular}{|c|l|}
\hline Property & Description \\
\hline L1 (Non-negativity) & The length of a system is nonnegative. \\
\hline L2 (Null value) & $\begin{array}{l}\text { The length of a system is } 0 \text { if the set of } \\
\text { elements which constitute the system is empty. }\end{array}$ \\
\hline $\begin{array}{c}\text { L3(Non Increasing } \\
\text { Monotonicity) }\end{array}$ & $\begin{array}{l}\text { Adding relationships between the elements of a } \\
\text { module m in a system does not increase the } \\
\text { length of the system. }\end{array}$ \\
\hline $\begin{array}{c}\text { L4 (Non Decreasing } \\
\text { Monotonicity) }\end{array}$ & $\begin{array}{l}\text { A system having modules } m 1 \text { and } m 2 \text { such that } \\
\text { they are represented by separate connected } \\
\text { components in the system. Adding } \\
\text { relationships from elements of } m 1 \text { to elements } \\
\text { of } m 2 \text { does not decrease the length of system. }\end{array}$ \\
\hline L5 (Merger) & $\begin{array}{l}\text { The length of a system made of union of two } \\
\text { disjoint modules } m 1 \text { and } m 2 \text { is equal to the } \\
\text { maximum of the lengths of } m 1 \text { and } m 2 .\end{array}$ \\
\hline
\end{tabular}

\section{INHERITANCE METRICS FOR EVALUATION}

This section presents the brief description of Depth of Inheritance Tree of a Class (DITC) metric and Class
Inheritance Tree (CIT) metric presented by Rajnish et al. [5-6] AND ICC (Inheritance Complexity of Class), and ICT (Inheritance Complexity of Tree) presented by Sandip et al. [3-4] for evaluation.

\section{A. Depth of Inheritance Tree Class (DITC) Metric}

The metric DITC for class inheritance hierarchy is measured in terms of sum of the attributes (Private, Protected, public and inherited) and Methods (Private, Protected, public and inherited) at each level [6]. The DITC metric of a class at each level is calculated as:

$$
C I T\left(C_{i}\right)=\Sigma
$$

Where,

$L E V_{i}=$ Attribute $\left(C_{i}\right)+\operatorname{Method}\left(C_{i}\right)$

$C_{i}=\mathrm{A}$ class in the $i^{\text {th }}$ level of class inheritance hierarchy.

Attribute $\left(C_{i}\right)=$ Count the total number of protected, private, public and inherited attributes within a class in the class inheritance hierarchy at each level.

Method $\left(C_{i}\right)=$ Count the total number of protected, private, public and inherited methods within a class in the class inheritance hierarchy at each level.

$L=$ Total height in the class inheritance hierarchy i.e. the maximum distance from the last node (last level in the class inheritance hierarchy) to the root node (first level in the class inheritance hierarchy), ignoring any shorter paths in case of multiple inheritance is used.

\section{B. Class Inheritance Tree (CIT) Metric}

The metric CIT is used to measure the class inheritance tree [6]. The primary purpose of this metric is to measure how class is inherited by multiple classes and how class inherits multiple classes at any level in the inheritance tree. $C I T$ is defined as follows:

$$
\operatorname{CIT}\left(C_{i}\right)=\sum_{i=1}^{l} \operatorname{CIN}(\mathrm{Ci})+\operatorname{COUT}(\mathrm{Ci})
$$

Where $C_{i}$ is the class at the $i^{\text {th }}$ level in the inheritance tree.

$\operatorname{CIN}\left(C_{i}\right)=1$ if $C_{i}$ is inherits multiple classes in the inheritance tree.

$=0$, otherwise.

COUT $\left(C_{i}\right)=1$ if $C_{i}$ is inherited by multiple classes in the inheritance tree.

$=0$, otherwise.

\section{Inheritance Complexity of Class (ICC)}

The metric ICC is given in [3] and is calculated as follows:

$$
I C C(C i)=\mathrm{M}(\mathrm{Ci})+\mathrm{A}(\mathrm{Ci})+I F(\mathrm{Ci})
$$




$$
I F(\mathrm{C} i)=\frac{\text { Noofclassesinheriteddirectlyby } C i}{1+\text { NoofclassesinheriteddirectlybyCi }}
$$

Where, Inheritance Complexity of Class $(I C C)$ is the metric value of a class of an inheritance tree.

$C_{i}=$ Classes at the $i^{\text {th }}$ level in an inheritance tree.

$A\left(C_{i}\right)=$ Count the number of attributes (protected, private, public and inherited attributes) at each level in an inheritance tree.

$M\left(C_{i}\right)=$ Count the number of methods (protected, private, public and inherited attributes) at each level in an inheritance tree.

\section{Inheritance Complexity of Tree (ICT)}

The metric ICT is given in [4] and is calculated as follows:

$$
I C T(\mathrm{C} i)=\frac{M(\mathrm{C} i)+A(\mathrm{C} i)+I F(\mathrm{C} i)}{N}
$$

Where

$$
I F(\mathrm{C} i)=\frac{\text { Noof classes inherited directly by } \mathrm{Ci}}{1+\text { Noof classes inherited directly by } \mathrm{Ci}}
$$

$N=$ Total number of classes in an inheritance tree.

\section{VIEWPOINTS AND PROJECTIONS}

The notion of viewpoints and projections was first introduced in [8] to aid classification of metrics. Viewpoint is the base at which the measurement is carried out and projections shows direction of interaction between the viewpoint and the portion of the program that is related to the measurement. Projections can be outward, inward or gross projections. At the end of this paper in Appendix $A$, shows the viewpoints and projections of other inheritance metrics presented by Krishna et al [7] and selected four metrics as mentioned in Section III. All these four metrics generates value for each class at each level, so view point of each metric is class level. But each class links to its parent classes in $D I T C$, so it has outwards projection. CIT metric is related to its parent classes and child classes, so it has gross projection. ICC and ICT metrics depends on all the classes in the system, so it is system viewpoint.

\section{THEORETICAL VALIDATION AGAINST BRIAND'S PROPERTY}

\section{A. Properties S1, S2, L1 and L2}

From the definition, it can be noted that for each metric there must be one positive or zero value. So, all the four metrics that have been described above is satisfied by Briand's size property $S 1, S 2$ and length property $L 1, L 2$.

\section{B. Properties $S 3$ and $L 5$}
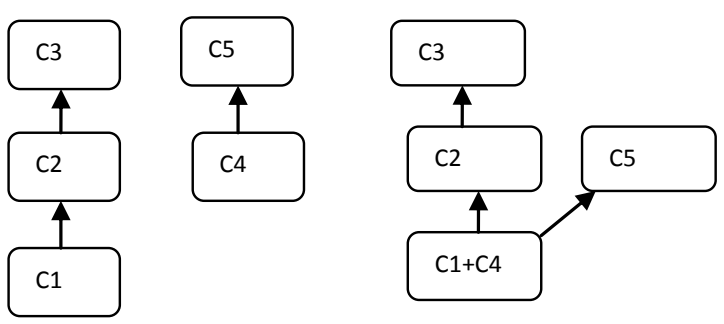

Fig.1: (a) Two disjoint module (b) After joining $\mathrm{C} 1$ and $\mathrm{C} 4$

Fig. 1 shows two disjoint modules, one have three levels ( $C 1$ is in first level and $C 3$ is in third level) and another have two levels ( $C 4$ is in first level and $C 5$ is in second level). After merging $C l$ and $C 4$, the class $C 1+C 4$ is in first level and $C 3$ is in third level. Suppose every class has one method and one attribute.

$$
\begin{gathered}
\operatorname{DITC}(C 1)=2 * 1=2 \\
\operatorname{DITC}(C 4)=2 * 1=2 \\
\operatorname{DITC}(C 1+C 4)=4 * 1=4 \\
\operatorname{DITC}(C 1)+\operatorname{DITC}(C 4)=2+2=4=\operatorname{DIT}(C 1+C 4)
\end{gathered}
$$

So, DITC metric satisfies size property $S 3$ and does not satisfy L5.

$C I T(C 1)=0$, because $C 1$ does not inherits multiple classes and it also does not inherited by multiple classes.

$C I T(C 4)=0$, because $C 4$ does not inherits multiple classes and it also does not inherited by multiple classes.

$C I T(C 1+C 4)=1$, because $C 1+C 4$ does not inherits multiple classes but it is inherited by multiple classes $C 2$ and $C 5$.

So, CIT metric doesn't satisfy property $S 3$ and also not satisfy L5.

$$
\begin{gathered}
\text { IF }(C 1)=1 / 2=0.5, \operatorname{IF}(C 4)=1 / 1=1 \text { and } I F(C 1+C 4) \\
=2 / 2=1 \\
\text { ICC }(C 1)=1+1+0.5=2.5 \\
I C C(C 4)=1+1+1=3 \\
\text { ICC }(C 1+C 4)=2+2+1=5
\end{gathered}
$$

So, ICC is not satisfied S3 and not satisfy L5.

$$
\begin{gathered}
\text { IF }(C 1)=1 / 2=0.5, \operatorname{IF}(C 4)=1 / 1=1 \text { and } \operatorname{IF}(C 1+C 4)= \\
2 / 2=1 \\
\operatorname{ICT}(C 1)=(1+1+0.5) / 3=0.833 \\
\operatorname{ICT}(C 4)=(1+1+1) / 2=0.67 \\
\operatorname{ICT}(C 1+C 4)=(2+2+1) / 4=1.25
\end{gathered}
$$

So, ICT is not satisfied S3 and not satisfy L5.

\section{Property L3}

A class viewpoint metric satisfies property $L 3$, as the metric does not use the relations among the methods and attributes. Adding new relations among the methods and attributes does not change the metric value. So, all the four inheritance metrics (DITC, CIT, ICC, and ICT) satisfied property $L 3$. 


\section{Property L4}

Consider Fig.2 that is slightly different from Fig. 1. The difference is that add one relationship between $C 1$ and $C 4$ in place of merging of $C 1$ and $C 4$.

DITC does not depend on relationship between two modules. So, DITC satisfy $L 4$.
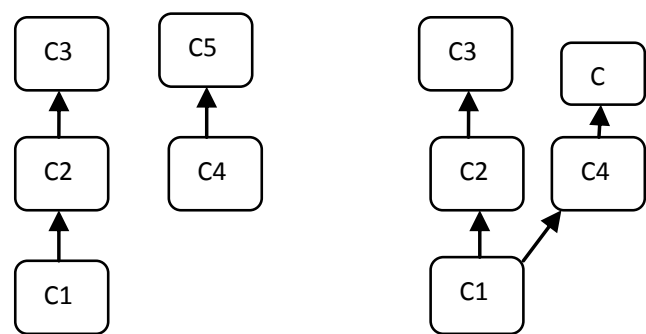

Fig.2: Two disjoint module and after adding relation between two disjoint connected modules

$C I T(C 1)=0$, when $C 1$ and $C 4$ are in different connected component.

But $C I T(C 1)=1$, when add relationship between $C 1$ and $C 4$. So, relationship may increase $C I T$ value but never decrease.

IF $(C 1)=1 / 2=0.5$, IF $(C 4)=1 / 1=1$ in different connected component, and $I F(C 1)=2 / 3=0.67$

Before adding relationship $I C C(C 1)=1+1+0.5=2.5$

After adding relationship $I C C(C 1)=1+1+0.67=2.67$

So, relationship may increase $I C C$ value but never decrease.

Before adding relationship ICT $(C 1)=(1+1+$ $0.5) / 3=0.833$

After adding relationship $\operatorname{ICT}(C 1)=(1+1+0.67) / 5$ $=0.534$

So, ICT is not satisfied $L 4$.

\section{CONCLUSION AND FUTURE SCOPE}

In this paper an attempt has been made to present a theoretical evaluation on Inheritance Metrics proposed by Rajnish et al [5-6] and Sandip et al [3-4] satisfy necessary properties of Briand et al [2]. At the end of this paper in Appendix B, summarizes the results found in this paper and another related paper [7]. The results show that DITC, CIT ICC, ICT metrics satisfies the necessary properties given by [2]. From Table 4 it is observed that out of 21 inheritance metrics, no metric satisfy all properties and all metric satisfy the property $S 1, L 1, S 2, L 2$, and $L 3$. Only Specialization Ratio and ICT do not satisfy L4. So S1, L1, $S 2, L 2, L 3$ and $L 4$ are the necessary properties to validate an inheritance metric. Four system viewpoint metrics (ICC, ICT, Specialization Ratio, and Reuse Ratio) does not satisfy property $S 3$ and L5. Fourteen metrics out of 21 (around 66\%) satisfy property $S 3$ and only 2 metric satisfy property $S 5$. In general, this firmly belief that $67 \%$ of the considered metrics are satisfied Briand's size property and 9\% metrics are satisfied Briand's length property. Only system view point metrics except $C I T$ are not satisfied by Briand property. So class view point metric is always satisfying the Briand property. All other metrics have to be revised to comply with the Briand [2] properties. Otherwise, use of these metrics as inheritance indicators is questionable.

The future scope focuses on some fundamental issues: (1) the work also points at a need for further work on the scope of existing validation properties and also on measurement concepts that are relatively less explored. (2) Empirically explore the relationships between the theoretical and empirical validation results.

\section{ACKNOWLEDGMENT}

The author would like to thanks the anonymous reviewers for their valuable comments and suggestions.

\section{REFERENCES}

[1] E. J. Weyuker, "Evaluating Software Complexity Measures", IEEE Trans. on Software Engineering, Vol.14, pp.1357-1365, 1998.

[2] L. C. Briand, S. Morasca, and V. R. Basili. "Propertybased software engineering measurement." IEEE Trans.on Software Eng., Vol.22, No. 1, 1996, pp.68-86.

[3] S. Mal and K. Rajnish. "Applicability of Weyuker's Property 9 to Inheritance Metric", International Journal of Computer Applications, Vol. 66, No.12. pp. 21-26, Published by Foundation of Computer Science, New York, USA, March 2013.

[4] S. Mal and K. Rajnish "New Quality Inheritance Metrics for Object-Oriented Design" International Journal of Software Engineering and Its Applications, Vol. 7, No. 6, pp. 185-200, Scopus (Elsevier) ISSN: 1738-9984, 2013

[5] K.Rajnish and Y. Singh. "An Empirical and Analytical View of New Inheritance Metric for Object-Oriented Design", International Journal of Computer Applications, Vol. 65, No.12, pp. 44-50, Published by Foundation of Computer Science, New York, USA, March 2013.

[6] K. Rajnish and V. Bhattacherjee, "Class Inheritance Metrics-An Analytical and Empirical Approach", INFOCOMP-Journal of Computer Science, Federal University of Lavras, Brazil, Vol. 7, No.3, pp. 25-34, 2008.

[7] G. S. Krishna, R. K. Joshi. "Inheritance Metrics: What do they Measure"MASPEGHI '10 Proceedings of the 4th Workshop on Mechanisms for Specialization, Generalization and inheritance. doi>10.1145/1929999.1930000. Published by ACM.

[8] P. Joshi and R. K. Joshi. "Microscopic coupling metrics for refactoring", Proceedings of the Conference on Software Maintenance and Reengineering, pp.145-152, 2006.

[9] K. Rajnish. "Class Complexity Metric to predict Understanability", International Journal of Information Enginering and Electronic Business, MECS Publishers, Vol. 6, No. 1, pp. 69-76, 2014.

[10] S.R. Chidamber and C.F. Kemerer, "A Metric Suite for Object-Oriented Design”, IEEE Trans. on Software Engineering, Vol. 20, No. 6, pp.476-493, 1994.

[11] B. Henderson-Sellers "Object Oriented Metrics: Measures of Complexity”, Prentice Hall PTR: Englewood Cliffs NJ, 1996.

[12] W. Li," Another metric suite for object-oriented programming", The Journal of Systems and Software Vol. 44, No. 2, pp.155-162, 1998.

[13] B. Kitchenham, S.L. Pfleeger, and N.E. Fenton, "Towards a framework for software measurement validation", IEEE Trans. On Software Engineering, Vol. 21, No. 12, pp. 929- 
944, 1995.

[14] P.D Tegarden, S.D. Sheetz, and D.E. Monarchi, "A software complexity model of OO Systems", Decision Support Systems, Vol. 13, No. 3, pp. 241-262, 1995.

[15] A. Lake, C. Cook, "Use of factor analysis to develop OOP software complexity metrics", Proceedings of the Annual Oregon Workshop on Software Metrics, Silver Falls OR. Oregon Center for Advanced Technology, April 10-12, 1994.

[16] M. Alshayeb and W. Li, "An Empirical Validation of Object-Oriented Metrics in Two Different Iterative Software Processes", IEEE Trans. on Software Engineering, Vol. 29, No. 11, pp. 1043-1049, 2003.

[17] K.K Agarwal, Y. Singh, A. Kaur and R. Malhotra "Empirical Study of Object Oriented Metrics", Journal of Object Technology, vol. 5, no. 8, Nov. - Dec. 2006, pp. $149-173$.

[18] K.K Agarwal, Y. Singh, A. Kaur and R. Malhotra, "Software Design Metrics for Object Oriented Software", Journal of Object Technology, vol. 6, no. 1, Jan. - Feb. 2007, pp. $121-138$.

\section{Author Profile}

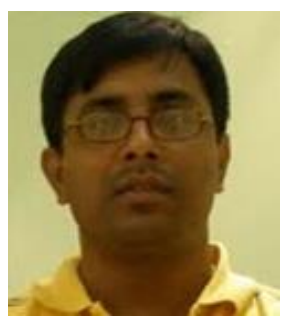

Dr. Kumar Rajnish is an Assistant Professor in the Department of Information Technology at Birla Institute of Technology, Mesra, Ranchi, Jharkahnd, India. He received his $\mathrm{PhD}$ in Engineering from BIT Mesra, Ranchi, Jharkhand, India in the year of 2009. He received his Master of Computer Application Degree from MMM Engineering College, Gorakhpur, State of Uttar Pradesh, India. He received his B.Sc Mathematics (Honours) from Ranchi College Ranchi, India in the year 1998. He has 30 International and National Research Publications. His Research area is Object-Oriented Metrics, Object-Oriented Software Engineering, Software Quality Metrics, Software Cost Estimation, Programming Languages, and Database System.

APPENDIX A METRICS WITH THEIR VIEWPOINTS AND PROJECTIONS

\begin{tabular}{|c|c|c|c|}
\hline Metric & Definition & Viewpoint & Projection \\
\hline LOC & Lines of Code & Program & Internal \\
\hline NOC & Number of Concrete Classes defined in a system & Class & Internal \\
\hline NOM & Number of Methods defined in a class & Class & Internal \\
\hline NOA & Number of Attributes defined in a class & Class & Internal \\
\hline SIZE2 & $\mathrm{NOM}+\mathrm{NOA}$ & Class & Internal \\
\hline NOK & Number of occurrences of a keywords in a program & Program & Internal \\
\hline NOAOP & Number of occurrences of a arithmetic operators in a program & Program & Internal \\
\hline $\begin{array}{c}\text { Class-Leaf Depth } \\
\text { (CLD) }\end{array}$ & Length of the path from the class to farthest leaf class & Nested & Inward \\
\hline Reuse Ratio (RR) & No. of superclasses / total no. of classes & System & Internal \\
\hline $\begin{array}{l}\text { Specialization Ratio } \\
\text { (SR) }\end{array}$ & No. of subclasses/no. of super-classes & System & Internal \\
\hline DIT & Depth of Inheritance of a class & Class & Nested Outward \\
\hline NOC & $\begin{array}{c}\text { Number of Children is the number of immediate subclasses } \\
\text { subordinated to a class }\end{array}$ & Class & Nested Inward \\
\hline Fandown & Number of subclasses of a class & Class & Nested Inward \\
\hline Fanup & Number of super classes of a class & Class & Nested Inward \\
\hline NIA & Number of Inherited Attributes in a class & Class & Nested Outward \\
\hline NIM & Number of Inherited Methods in a class & Class & Nested Outward \\
\hline NoVM & Number of Overridden Methods in a class & Class & Nested Gross \\
\hline DITC & Mentioned in Section III & Class & Outward \\
\hline CIT & Mentioned in Section III & Class & Gross \\
\hline ICC & Mentioned in Section III & System & Internal \\
\hline ICT & Mentioned in Section III & System & Internal \\
\hline
\end{tabular}


APPENDIX B THEORETICAL VALIDATION RESUlts OF INHERITANCE METRICS AGAINST BRIAND’s SiZE AND LENGTH PROPERTy [V: METRICS SATISFIES PROPERTIES $\quad \times$ : METRICS DOES NOT SATISFY PROPERTIES]

\begin{tabular}{|c|c|c|c|c|c|c|}
\hline Metric & $S 1, L 1$ & $S 2, L 2$ & $S 3$ & L3 & $L 4$ & L5 \\
\hline $\mathrm{LOC}$ & $\sqrt{ }$ & $\sqrt{ }$ & $\sqrt{ }$ & $\sqrt{ }$ & $\sqrt{ }$ & $\times$ \\
\hline $\mathrm{NOC}$ & $\sqrt{ }$ & $\sqrt{ }$ & $\sqrt{ }$ & $\sqrt{ }$ & $\sqrt{ }$ & $x$ \\
\hline NOM & $\sqrt{ }$ & $\sqrt{ }$ & $\sqrt{ }$ & $\sqrt{ }$ & $\sqrt{ }$ & $x$ \\
\hline NOA & $\sqrt{ }$ & $\sqrt{ }$ & $\sqrt{ }$ & $\sqrt{ }$ & $\sqrt{ }$ & $x$ \\
\hline SIZE2 & $\sqrt{ }$ & $\sqrt{ }$ & $\sqrt{ }$ & $\sqrt{ }$ & $\sqrt{ }$ & $x$ \\
\hline $\mathrm{NOK}$ & $\sqrt{ }$ & $\sqrt{ }$ & $\sqrt{ }$ & $\sqrt{ }$ & $\sqrt{ }$ & $x$ \\
\hline NOAOP & $\sqrt{ }$ & $\sqrt{ }$ & $\sqrt{ }$ & $\sqrt{ }$ & $\sqrt{ }$ & $x$ \\
\hline Class-Leaf Depth (CLD) & $\sqrt{ }$ & $\sqrt{ }$ & $x$ & $\sqrt{1}$ & $\sqrt{ }$ & $\sqrt{ }$ \\
\hline Reuse Ratio (RR) & $\sqrt{ }$ & $\sqrt{ }$ & $x$ & $\sqrt{ }$ & $\sqrt{ }$ & $x$ \\
\hline Specialization Ratio (SR) & $\sqrt{ }$ & $\sqrt{ }$ & $x$ & $\sqrt{ }$ & $x$ & $x$ \\
\hline DIT & $\sqrt{ }$ & $\sqrt{ }$ & $x$ & $\sqrt{ }$ & $\sqrt{ }$ & $\sqrt{ }$ \\
\hline $\mathrm{NOC}$ & $\sqrt{ }$ & $\sqrt{ }$ & $\sqrt{ }$ & $\sqrt{ }$ & $\sqrt{1}$ & $x$ \\
\hline Fandown & $\sqrt{ }$ & $\sqrt{ }$ & $\sqrt{ }$ & $\sqrt{ }$ & $\sqrt{ }$ & $x$ \\
\hline Fanup & $\sqrt{ }$ & $\sqrt{ }$ & $\sqrt{ }$ & $\sqrt{ }$ & $\sqrt{ }$ & $x$ \\
\hline NIA & $\sqrt{ }$ & $\sqrt{ }$ & $\sqrt{ }$ & $\sqrt{ }$ & $\sqrt{ }$ & $x$ \\
\hline NIM & $\sqrt{ }$ & $\sqrt{ }$ & $\sqrt{ }$ & $\sqrt{1}$ & $\sqrt{ }$ & $x$ \\
\hline NoVM & $\sqrt{ }$ & $\sqrt{ }$ & $\sqrt{ }$ & $\sqrt{ }$ & $\sqrt{ }$ & $x$ \\
\hline DITC & $\sqrt{ }$ & $\sqrt{ }$ & $\sqrt{ }$ & $\sqrt{ }$ & $\sqrt{ }$ & $x$ \\
\hline CIT & $\sqrt{ }$ & $\sqrt{ }$ & $x$ & $\sqrt{ }$ & $\sqrt{ }$ & $x$ \\
\hline ICC & $\sqrt{ }$ & $\sqrt{ }$ & $x$ & $\sqrt{ }$ & $\sqrt{ }$ & $x$ \\
\hline ICT & $\sqrt{ }$ & $\sqrt{ }$ & $x$ & $\sqrt{ }$ & $x$ & $x$ \\
\hline
\end{tabular}

How to cite this paper: Kumar Rajnish,"Theoretical Validation of Inheritance Metrics for Object-Oriented Design against Briand's Property", IJIEEB, vol.6, no.3, pp.28-33, 2014. DOI: 10.5815/ijieeb.2014.03.05 